\title{
Acute ST-segment Elevation Myocardial Infarction in Kommerell's Diverticulum with a Right-sided Aortic Arch: A Case Report
}

\author{
Kaito Abe ${ }^{1,2}$, Takemi Kusano ${ }^{1}$, Ryota Nakajima ${ }^{1}$, Koki Sogame ${ }^{1,2}$, Hidetoshi Fukui ${ }^{1}$, Moto Shimada ${ }^{1,2}$, \\ Hiroshi Doi ${ }^{1,2}$, Goro Endo ${ }^{1}$, Kaori Kanbara ${ }^{1,2}$, Tomoaki Ishigami ${ }^{2}$, Kouichi Tamura ${ }^{2}$ and Jun Okuda ${ }^{1,2}$
}

\begin{abstract}
Kommerell's diverticulum is a very rare congenital aortic arch anomaly with an estimated incidence of 0.04$0.1 \%$. Few studies have reported on acute ST-segment elevation myocardial infarction in patients with Kommerell's diverticulum on the right-sided aortic arch. The anatomical anomaly makes coronary artery catheterization difficult. If such a case is suspected during the patient examination before coronary artery revascularization, the appropriate arterial approach site should be carefully considered for revascularization as early as possible.
\end{abstract}

KEY WORDS: Kommerell's diverticulum, right-sided aortic arch, ST-elevation myocardial infarction

\section{Introduction}

Kommerell's diverticulum was first reported by Dr. Kommerell in 1936 as a dilatation of the aorta ${ }^{1)}$. It is mostly found at the origin of an aberrant subclavian artery as a bulblike swelling and is seen in $20 \%-60 \%$ of both right- and left-sided aortic $\operatorname{arches}^{1}{ }^{1}$. Approximately half of the right-sided aortic arch cases are associated with an abnormal origin of the left subclavian artery $^{2)}$. Kommerell's diverticulum with a right-sided aortic arch is rare, with an incidence between 0.04 and $0.1 \%^{2,3)}$. There are few reports of acute ST-segment elevation myocardial infarction (STEMI) in Kommerell's diverticulum with a right-sided aortic arch. Early revascularization of myocardial ischemia can improve patient prognosis ${ }^{4,5}$. A radial artery approach is recommended rather than a femoral artery approach owing to the lower rates of complications in acute coronary syndrome (ACS) $)^{4}$. However, early revascularization using a catheter can be difficult due to the anatomical anomaly, as seen in the case described below. It is especially important to select the appropriate arterial approach site for the patient's anatomical anomaly. The patient

\footnotetext{
${ }^{1}$ Department of Cardiology, Omori Red Cross Hospital, 4-30-1 Chuo, Ota-ward, Tokyo, 143-8527, Japan, ${ }^{2}$ Department of Medical Science and Cardiorenal Medicine, Yokohama City University Graduate School of Medicine

E-mail: kaitoabemed@gmail.com

Received: November 26, 2020; Accepted: February 4, 2021

J-STAGE Advance Published: June 3, 2021

doi: 10.7793/jcad. 20-00031

(C) The Japanese Coronary Association
}

provided written informed consent to publish this case report, and the publication procedure was approved by the Ethics Committee at our hospital (No. 20-12.2020/7/21).

\section{Case report}

A 57-year-old man presented at a nearby hospital with a complaint of severe substernal chest pain during sleep ; ST-elevation acute myocardial infarction was suspected. He had a history of dyslipidemia and smoking (20 cigarettes per day). At the time of admission, physical examination revealed a blood pressure of $149 / 107 \mathrm{mmHg}$, pulse rate of 80 beats/min, lungs clear to auscultation, a regular respiratory rate, and no murmurs or gallops. The initial ECG (Fig. 1a) revealed ST-elevation of $1 \mathrm{~mm}$ or greater $(0.1 \mathrm{mV})$ in leads V1 through V5. The laboratory findings showed elevated cardiac troponin I: $881.3 \mathrm{pg} / \mathrm{mL}$, creatine kinase (CK): $363 \mathrm{IU} / \mathrm{L}, \mathrm{MB}$ isoenzyme of CK: $21 \mathrm{IU} / \mathrm{L}$, and low-density lipoprotein: $182 \mathrm{mg} / \mathrm{dL}$. However, chest radiographs did not reveal any significant findings apart from the right-sided aortic arch (Fig. 1b). Transthoracic echocardiography revealed the asynergy of the anterior and apical segments ; the visual estimated ejection fraction was preserved at $55 \%$, and no significant valvular disease was observed in the emergency department. These findings suggested acute STEMI. Emergency coronary angiography was performed using the right radial arterial approach with a catheter (5Fr, JL40, GOODTEC ANGIOGRAPHIC CATHETER, Goodman Corporation, Japan) to the left coronary artery. Torque transmission in the ascending aorta was difficult during catheterization due to the anatomical anomaly. The catheter did not reach the left coronary artery and was pushed up for engagement. Coronary angiography revealed a to- 


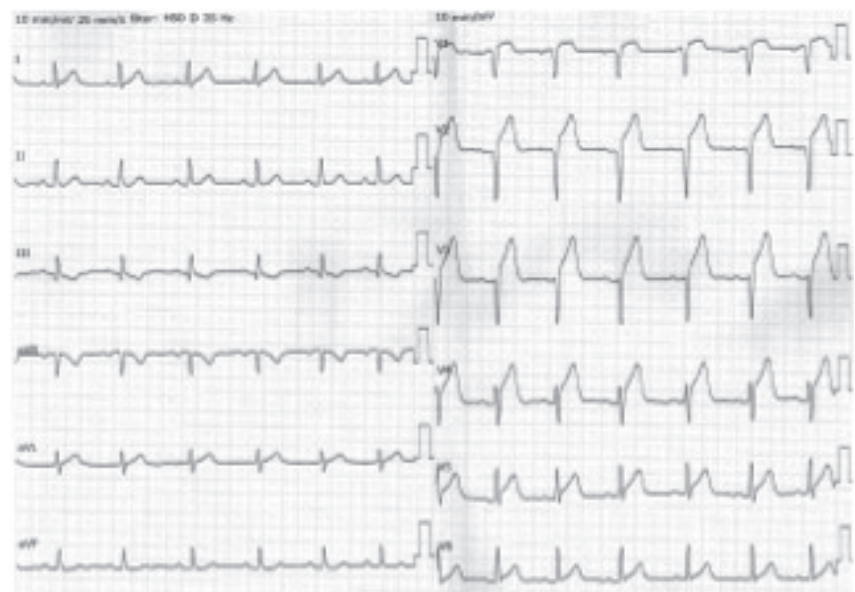

(a)

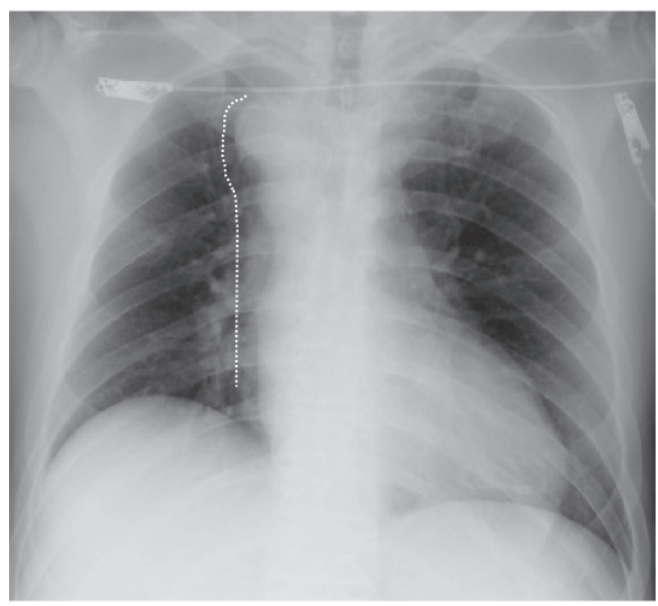

(b)

Fig. 1

a: Electrocardiogram on admission showing ST elevation in leads. (V1-V5)

b: Chest radiograph (AP view) showing the right-sided aortic arch. (White dotted line)

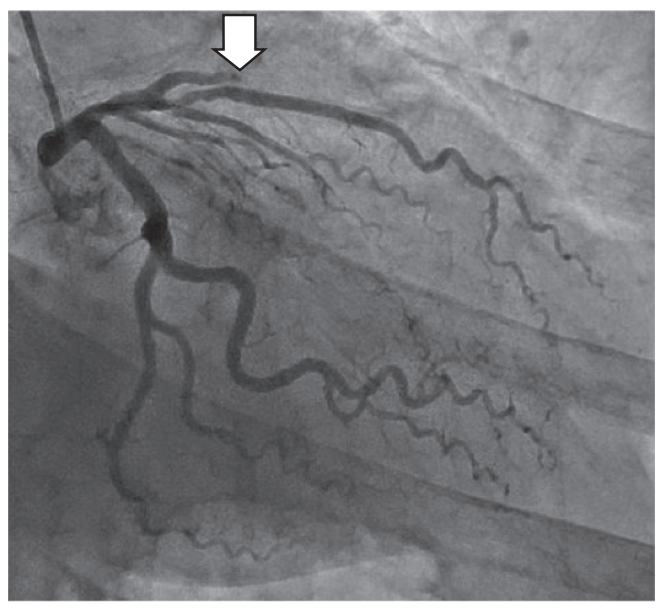

(a)

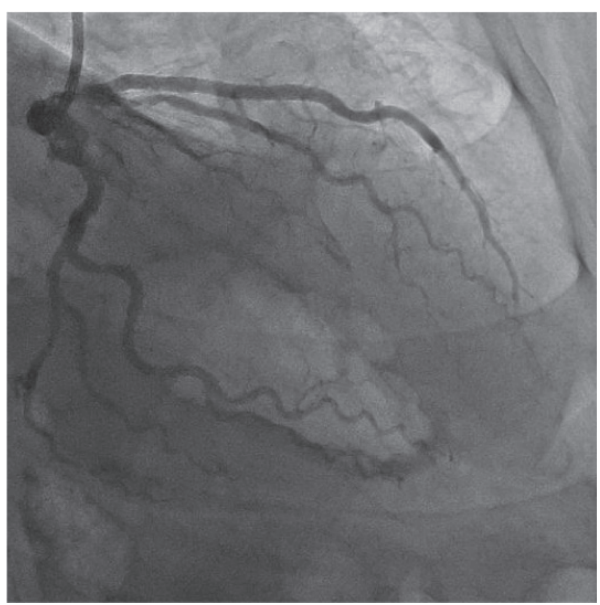

(b)

Fig. 2

a: Coronary angiogram showing total occlusion at the proximal lesion of the left anterior descending artery. (RAO $30^{\circ}$, CAU $25^{\circ}$ ) (White arrow)

b: Coronary angiogram showing revascularization of the culprit lesion after the implantation of a drug-eluting stent. (RAO $\left.40^{\circ}, \mathrm{CAU} 40^{\circ}\right)$

right anterior oblique view: RAO, caudal view: CAU

tal occlusion of the left anterior descending artery (Fig. 2a). Emergency percutaneous coronary intervention (PCI) was performed. Since the diagnostic catheter (JL40) engaged the left coronary artery by being pushed up, a back-up type therapeutic catheter (7Fr SPB3.5, SPB4.0, ASAHI Hyperion Guiding catheter, (CASAHI INTECC J-Sales, INC, Japan) was first selected. However, catheter operation was difficult in engaging the left coronary artery. By changing the guide wire from the standard type (Radifocus Guidewire M Standard Type 0.035”, TERUMO, Corporation, Japan) to a hard type (Goodtec Spring Guidewire 0.035", Goodman Corporation, Japan) in order to improve torque transmission and operability, and changing the therapeutic catheter from (SPB3.5, SPB4.0) to (7Fr JL40, ASAHI Hyperion Guiding catheter, (CASAHI INTECC J-Sales, INC, Japan), the therapeutic catheter could engage the left coronary artery. Thrombus aspiration (7Fr Thrombuster III GR, KANEKA MEDIX CORPORATION, Japan) was performed, and a drug-eluting stent (Ultimaster Tansei 2.75*33 mm, TERUMO, Corporation, Japan) was placed; the vessel was successfully re-canalized (Fig. 2b). Computed tomography (CT) revealed a Kommerell's diverticulum from the right-sided aortic arch with an aberrant left subclavian artery after catheter intervention 


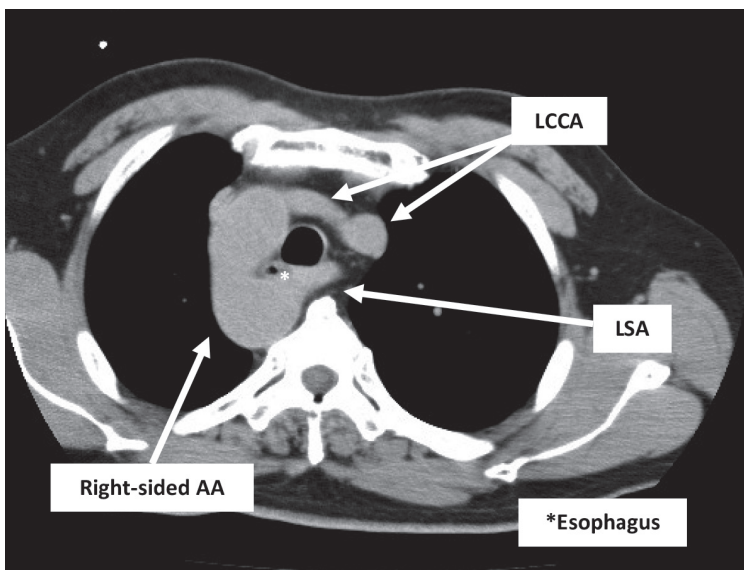

(a)

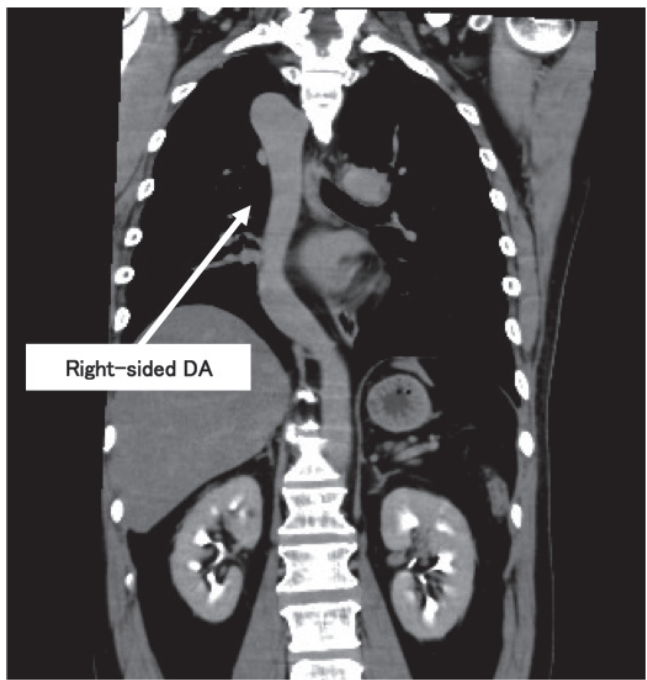

(c)

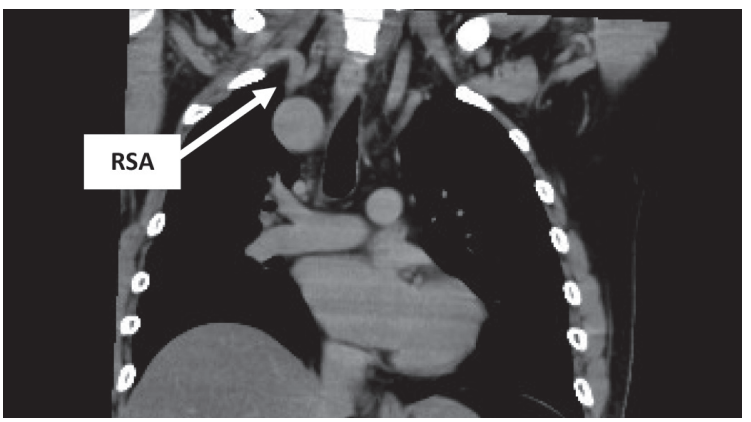

(e)

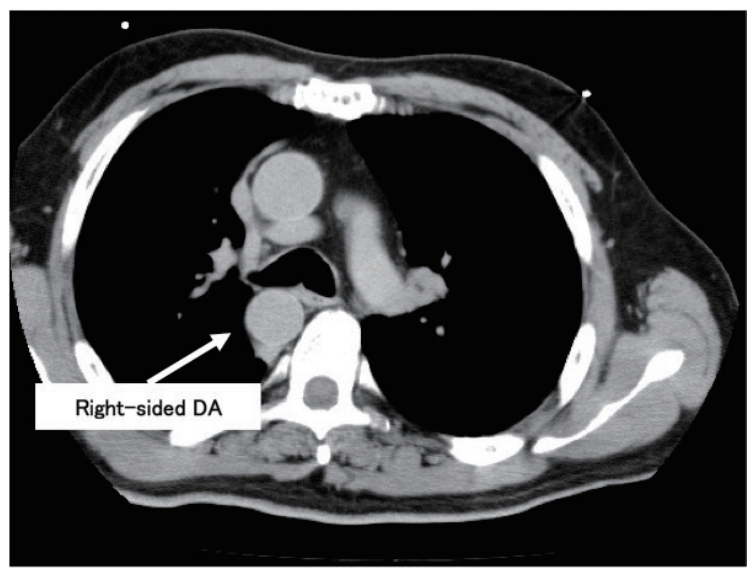

(b)

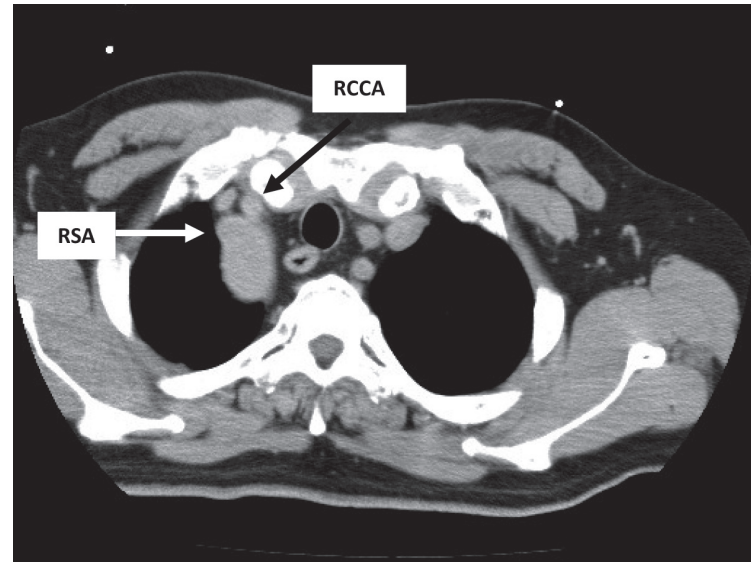

(d)

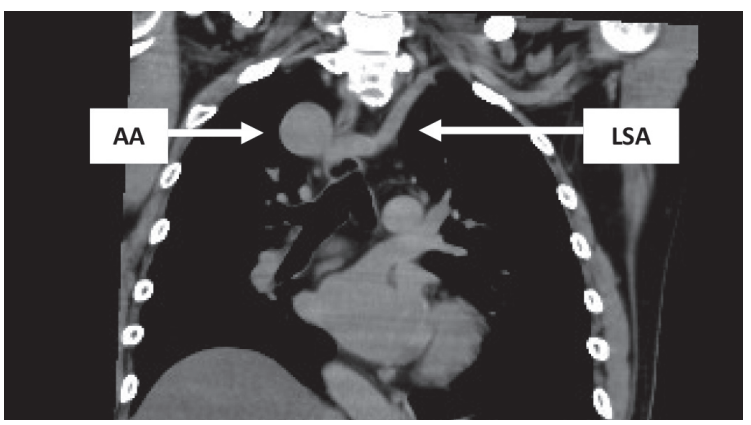

(f)

Fig. 3 Computed tomography (CT) showing the right-sided aortic arch and an aberrant left subclavian artery from Kommerell's diverticulum.

a: LCCA, LSA, Right-sided AA, Esophagus. CT axial view

b: Right-sided DA. CT axial view

c: Right-sided DA. CT coronal view

d: RCCA, RSA. CT axial view

e: RSA. CT coronal view

f: AA, LSA. CT coronal view

AA: aortic arch, DA: descending aorta, LCCA: left common carotid artery, LSA: left subclavian artery, RCCA: right common carotid artery, RSA: right subclavian artery 
(Figs. 3a, b, and c). First the left common carotid artery branched off from the ascending aorta, passed through the anterior bronchus and rose to the neck, then the right common carotid artery branched independently (Figs. 3a and d). The right subclavian artery ran from the aortic arch to the outside of the thorax at an acute angle. (Figs. 3d and e). Finally, the left subclavian artery was bifurcated (Figs. 3a and f). The door-to-balloon time was 96 min, and peak CK was 4,075 IU/l. The patient was discharged 8 days after admission with a good clinical course.

\section{Discussion}

Kommerell's diverticulum has three distinct types based on their location: in the left-sided aortic arch, in the right-sided aortic arch, and at the aortic-ductal junction. The left-sided diverticulum is associated with an aberrant right subclavian artery, while the right-sided aortic arch with an aberrant left subclavian artery $^{3)}$. The right-sided aortic arch itself can also be classified into three types according to the arterial branches from the aortic arch: Type 1, with mirror image branching of the major arteries ; Type 2, with an aberrant left subclavian artery ; and Type 3, with an isolated left subclavian artery connected from the pulmonary artery ${ }^{2}$. This case is classified as an aortic arch Type 2.

The aberrant subclavian arteries are found in the contralateral arm after passing the posterior esophagus $(80 \%)$ as in this case (Fig. 3a). Less frequently, these arteries can pass between the esophagus and the trachea $(15 \%)$ or anterior to the trachea $(5 \%)^{1)}$. Adult patients with an aberrant artery can present with dysphagia, a blood pressure difference in the upper limbs, or chest pain in about five percent of the cases ${ }^{1)}$. A right-sided aortic arch with an aberrant left subclavian artery is twice as prevalent among men as it is among women ${ }^{1)}$. Congenital heart disease has been reported to be associated with 5-10\% of patients with Type 2 Kommerell's diverticulum and $75-85 \%$ of those with Type 1 and Type 3 Kommerell's diverticulum ${ }^{2}$.

Surgical treatment can be considered depending on the anatomical structure, size, and symptoms of each Kommerell's diverticulum $^{1)}$. There is one case report of STEMI with the normal left-sided aortic arch associated with an aberrant right subclavian artery ${ }^{6}$. However, it is a case without coronary artery stenosis on angiography, and its pathology was unclear. This is the first reported case of an acute STEMI in Kommerell's diverticulum with a right-sided aortic arch. Since in this case it was difficult to perform PCI due to the anatomical anomaly, it seems to be an important case when considering future treatment strategies.

In this case, the patient was admitted with acute STEMI and chest radiography suggested a right-sided aortic arch. Despite the anatomical difficulties of using a right radial artery approach for the right-sided aortic arch, emergency coronary angiography was nonetheless performed from the right radial artery in order

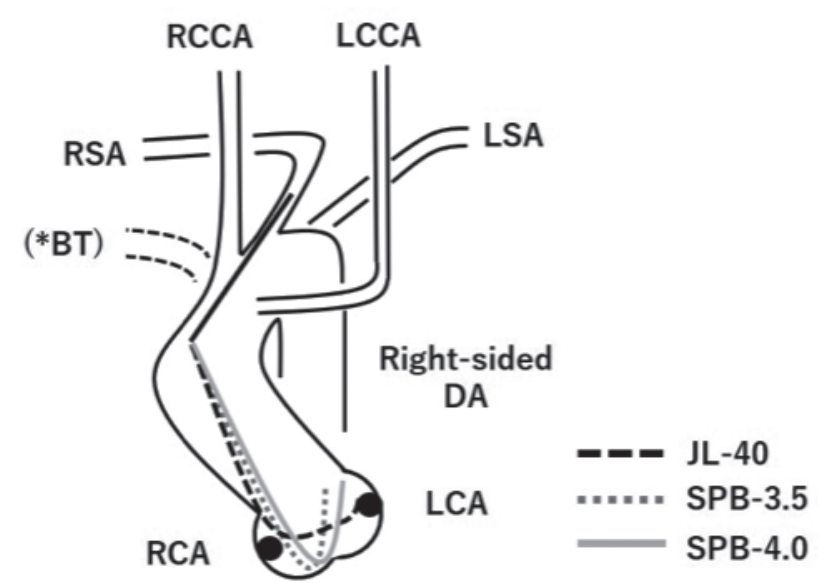

Fig. 4 Schema of relationship between anatomical structure and catheter position.

*The dotted brachiocephalic trunk indicates a common anatomical structure. (Dotted line)

BT: brachiocephalic trunk, DA: descending aorta, LCA: left coronary artery, LCCA: left common carotid artery, LSA: left subclavian artery, RCA: right coronary artery, RCCA: right common carotid artery, RSA: right subclavian artery

to attempt reperfusion as early as possible and in a minimally invasive manner. Because early revascularization of myocardial ischemia correlates with patient prognosis, a door-to-balloon time within $90 \mathrm{~min}$ is targeted ${ }^{5}$. However, due to the anatomical abnormality of the right-sided aortic arch with Kommerell's diverticulum (Type 2) and the right subclavian artery running from the aortic arch to the outside of the thorax at an acute angle, the catheter operability due to factors such as torque transmission was poor, and it was difficult for the catheter to engage in the coronary artery (Fig. 4). The door-to-balloon target time had exceeded $90 \mathrm{~min}$. If the left radial artery is selected for the approach site, it can be expected that the catheter operation will be more complicated than the right radial artery approach (Fig. 3a).

A radial artery approach is generally recommended in $\mathrm{ACS}^{4)}$. However, if a right-sided aortic arch is suspected during the examination before emergency coronary arterial angiography in patients with STEMI, the patient may suffer from Kommerell's diverticulum with a right-sided aortic arch and therefore the operator should avoid the left radial artery approach. The right radial artery would be possible; however, if manipulation of the guiding catheter is difficult, an alternative femoral artery access should be considered as it is important to revascularize the occluded culprit coronary as early as possible. It may be desirable to perform plane or contrast-enhanced CT before PCI, except if it would delay reperfusion therapy. If the manipulation of the guiding catheter is difficult, it may be also desirable to perform aortography with a pigtail catheter during PCI to select an appropriate approach site that suits the patient's anatomical anomaly. 


\section{Conclusion}

We have presented a rare case of acute STEMI in Kommerell's diverticulum with a right-sided aortic arch. If its presence is suspected in an examination before coronary arterial revascularization, the choice of arterial approach site should be carefully considered to avoid a delay in revascularization.

\section{Conflicts of interest}

All authors declare that they have no conflicts of interest.

\section{References}

1) Tanaka A, Milner R, Ota T: Kommerell's diverticulum in the current era: A comprehensive review. Gen Thorac Cardiovasc Surg 2015; 63: $245-259$

2) Cinà CS, Althani H, Pasenau J, et al: Kommerell's diverticulum and right-sided aortic arch: A cohort study and review of the literature.
J Vasc Surg 2004; 39: 131-139

3) Salomonowitz E, Edwards JE, Hunter DW, et al: The three types of aortic diverticula. AJR Am J Roentgenol 1984; 142: 673-679

4) Kimura K, Kimura T, Ishihara M, et al: JCS 2018 guideline on diagnosis and treatment of acute coronary syndrome. Circ J 2019; 83: 1085-1196

5) Hunt SA, Baker DW, Chin MH, et al: ACC/AHA guidelines for the evaluation and management of chronic heart failure in the adult: Executive summary a report of the American College of Cardiology/ American Heart Association task force on practice guidelines (Committee to revise the 1995 guidelines for the evaluation and management of heart failure): Developed in collaboration with the International Society for Heart and Lung Transplantation; Endorsed by the Heart Failure Society of America. Circulation 2001; 104: 2996-3007

6) Alirhayim Z, Qureshi W, Shafiq A, et al: Aortic arch variant presenting as an acute ST elevation myocardial infarction. BMJ Case Rep 2013: bcr2012007962 